\title{
Fracture of colloidal single-crystal films fabricated by controlled vertical drying deposition
}

\author{
He Cao, ${ }^{1}$ Ding Lan, ${ }^{1, *, \dagger}$ Yuren Wang, ${ }^{1, *, \$}$ Alex A. Volinsky, ${ }^{2}$ Li Duan, ${ }^{1}$ and Heng Jiang ${ }^{1}$ \\ ${ }^{1}$ Key Laboratory of Microgravity (National Microgravity Laboratory), Institute of Mechanics, Chinese Academy of Sciences, \\ Beijing 100190, China \\ ${ }^{2}$ Department of Mechanical Engineering, University of South Florida, Tampa, Florida 33620, USA
}

(Received 6 April 2010; published 2 September 2010)

\begin{abstract}
Controlled vertical drying deposition method was used to make high-quality single crystal close-packed colloidal films formed of different radii polystyrene latex spheres on glass substrates coming from a low concentration water suspension $(0.1 \%$ volume fraction). Regardless of the spheres radii the film thickness was about 6.3 microns. However, cracks destroyed the crystalline film structure during the colloidal film growth. The effect of particle radius (85-215 $\mathrm{nm}$ range) on film cracking was systematically studied using in situ optical fracture monitoring. Primary parallel cracks run along the vertical growth direction, later followed by secondary branched cracks in-between the primary cracks due to residual water evaporation. Quantitative theoretical relationship between the cracks spacing and particles radius was derived and shows good agreement with experimental observations. Normalized cracks spacing is related to a reciprocal ratio of the dimensionless particle radius.
\end{abstract}

DOI: 10.1103/PhysRevE.82.031602

PACS number(s): 81.10.Dn, 81.40.Np

\section{INTRODUCTION}

Over the last few years colloidal crystals have attracted researchers' attention due to their potential applications in photonics [1-4]. Meanwhile there are many methods [5-9] that could be used to form colloidal crystals, such as sedimentation and controlled vertical drying deposition (CVDD) [5]. Among these methods, CVDD has lately become one of the most favorable, since it is capable of precisely controlling the film crystal structure and producing high-quality colloidal crystals. This method can be easily realized by placing the substrate either vertically or tilted in a dilute suspension of monodispersed particles. As the liquid evaporates, a largearea face-centered-cubic (FCC) crystalline film is deposited on the substrate at the contact line with the suspension meniscus. The crystalline film is formed due to interactions among the colloidal particles. These interactions include capillary forces, liquid surface tension, tensile stress, gravitational force, electrostatic repulsion, and van der Waals forces [10-13]. Cracks emerging during colloidal film deposition destroy its periodic structure [14-16]. Unfortunately, film cracks cannot be completely avoided at this point and become a bottle neck of CVDD colloidal crystals photonic applications. It is important to understand the cracking mechanism for getting crack-free colloid crystals.

For a long time, cracking in various drying systems has been investigated, including wet clays, ceramic and latex films [17-20]. Model colloidal dispersions were usually used by researchers to study the cracking mechanism. Recently, a fundamental understanding of the cracking mechanism has emerged [21-23]: particles deformation caused by solvent evaporation from the film formed of close-packed particles must be responsible for the cracks formation under given

\footnotetext{
*Author to whom correspondence should be addressed.

†landing@imech.ac.cn

†wangyr@imech.ac.cn
}

conditions, for example, in the drying process of the film fabricated by CVDD. Deformation theories of the spheres in contact were derived from Frenkel model [24], Herz theory [25], Johnson-Kendall-Roberts (JKR) [26] or DerjaguinMuller-Toporov (DMT) approximations [27]: instead of a spot contact, face contact occurred when two touching spheres were compressed by external forces. Then, a relationship between the external force and the strain was obtained based on the JKR theory results. Recently, Routh and Russel [28] considered viscoelastic deformation of particles pair caused by both interfacial tension and an external force, obtained a macroscopic stress-strain relationship and then Tirumkudulu and Russel [21] used energy balance analysis to predict crack dynamics: they utilized Griffith's criterion for equilibrium crack propagation along with the stress-strain relationship mentioned above to predict the critical stress $\sigma_{0}$ for nucleation of an isolated crack and the crack spacing for multiple cracks.

Meanwhile, cracking during drying of a wet film has been observed in diverse systems, mainly by two ways: one is the different shape "droplet" drying experiment [19-22,29]; and the other is the directional drying experiment $[20,23,30]$. These drying experiments undergo three stages: concentration, gel formation, and consolidation processes. The outcomes strongly depend on the original particles concentration in suspension. Large-area films only form at high particle concentrations $(20-50 \%$ volume fraction $[19,21,23,30])$. When particle concentration decreases, diversiform drying patterns are formed, including "ring" [22,31] and "flower" patterns [29]. Also, concentration changes result in not-uniform film structure, where particles are no longer close-packed in these experiments. However, our CVDD experimental system of colloidal spheres is different: (a) it is capable of growing large-scale crystals from low particle concentration suspensions ( $0.1 \%$ volume fraction); (b) without gel formation, the colloidal particles selfassemble to be a film by capillary forces and micro flow at the vicinity of the solvent menisci; (c) a film with uniform thickness is formed of close-packed spheres. Thus, it is dif- 


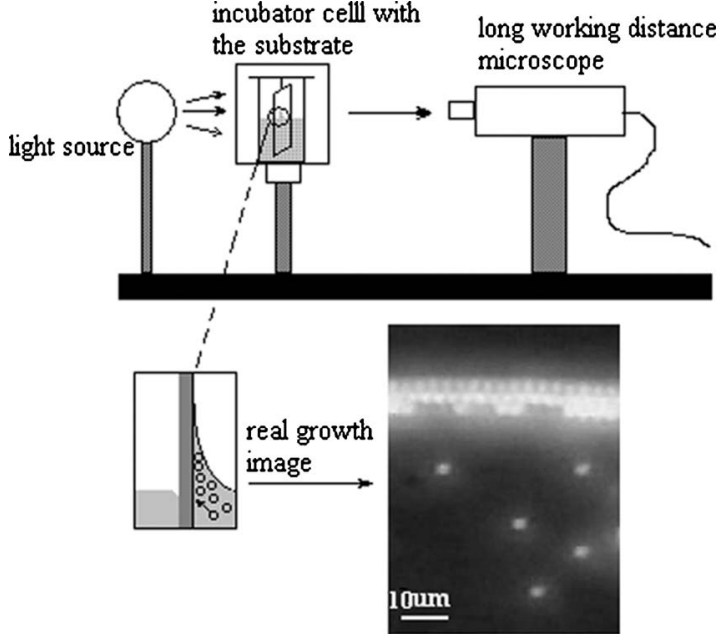

FIG. 1. A schematic of the experimental setup. A magnified schematic of the observed region is shown at the vicinity of the meniscus, as well as the image observed at the initial growing stage. Large colloid spheres with the diameter of 3 micrometers were used in the observation of the initial assembly process.

ficult to explain results obtained in this paper only by utilizing the above-mentioned crack-forming theories.

In this article, we report a real-time observation of crack patterns formation in the controlled vertical drying deposition experiment, focusing on the effect of the particle radius on the cracks spacing. For longitudinal cracks, the former conclusions along with the crystal fracture theory were utilized to provide theoretical analysis of the film fracture. Theoretical analysis of the relationship between the cracks spacing and the particle radius shows good experimental correlation.

\section{EXPERIMENTAL DETAILS}

Figure 1 is the schematic diagram of experimental setup. A magnified schematic of the observed region is shown, as well as the image observed at the initial growth stage. To have a comprehensive understanding of the assembly process, large colloid spheres with the diameter of $3 \mu \mathrm{m}$ were used for the observation of the initial assembly process. Cracks formation process by CVDD was observed in a transparent glass cell. Polystyrene latex spheres (from Duke Scientific Corporation, California, USA) with $85,110,150,180$, and $215 \mathrm{~nm}$ radii were used in this work. Polystyrene spheres were monodispersed in Milli-Q water with $0.1 \%$ volume fraction. The suspension was transferred into a semicylindrical glass growth cell. A $4 \times 1.5 \mathrm{~mm}$ rectangular, $1 \mathrm{~mm}$ thick glass substrate was inserted vertically into the growth cell.
To guarantee good water wetting, the glass substrate and the growth cell walls were cleaned with detergent, followed by immersion in chromic acid for about $24 \mathrm{~h}$. Cleaned glass plates were kept in Milli-Q water for about $8 \mathrm{~h}$, and dried naturally in the clean room environment before experiments. Glass growth cell was kept at $50{ }^{\circ} \mathrm{C}$ during the experiment. Mean water evaporation rate was about $0.17 \mathrm{~g} /$ hour. The observation was focused on the region in the vicinity of the suspension meniscus. A long working distance optical microscope was used to carry out experimental observations. Colloid self-assembly process was recorded with a digital video recorder connected to the microscope camera.

\section{RESULTS AND DISCUSSION}

The colloidal crystal formation by CVDD is mainly controlled by capillary forces acting among the particles and convection at the vicinity of the liquid meniscus $[32,33]$ with a high Péclet number. Figure 2(a) is a schematic diagram of the film formation by CVDD. During the film formation process colloidal particles self-assemble at the vicinity of the contact line. The colloidal particles self-assemble to be a uniform film directly on the substrate (Fig. 1) and the film does not undergo a gel formation process. Further evaporation causes the liquid meniscus at the top layer of the particle network to exert compressive capillary forces on the particle network. The film binds to the substrate and resists deformation in the transverse direction giving rise to transverse tensile stress. Cracks in the film form and release transverse tensile stress. This is different compared with Lee and Routh's experiments [22] and C. Allain and L. Limat's experiments [18]. Because of the high initial concentration, gel forms as solvent evaporates in their experiments. Capillary tension induces water drainage to prevent air exposure of the particles near the meniscus leading to gel shrinkage. Finally films are formed by gel shrinkage and particles diffusion with further water evaporation often characterized by a low Péclet number. The gel sticks to the cell glass plates which results in high stresses, the origin of the cracks formation. Also, concentration variations during the drying process prevent uniform crystalline film formation.

The film growth of CVDD could be divided into three regions [shown schematically in Fig. 2(a)]: dried region I, partially dried region II, and saturated region III. Figure 2(b) is the real time observation of the colloidal crystal formation (110 $\mathrm{nm}$ radius particles). Cracks form in the partially dried film regions, and the crack-free region is below the saturated region. Unfortunately, the film is so thin that it is very difficult to distinguish between the partially dried and the saturated regions. Figures 2(b) and 2(c) show cracks formation during the colloidal crystal growth with $110 \mathrm{~nm}$ radius par-

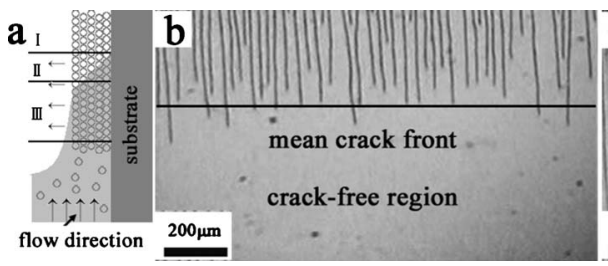

FIG. 2. (a) Schematic drawing of the film growth: I dried region; II partially dried region; III saturated region; (b) optical image of the colloidal crystal formation $(R=110 \mathrm{~nm})$; (c) optical image taken $2 \mathrm{~h}$ after $\mathrm{b}$. 


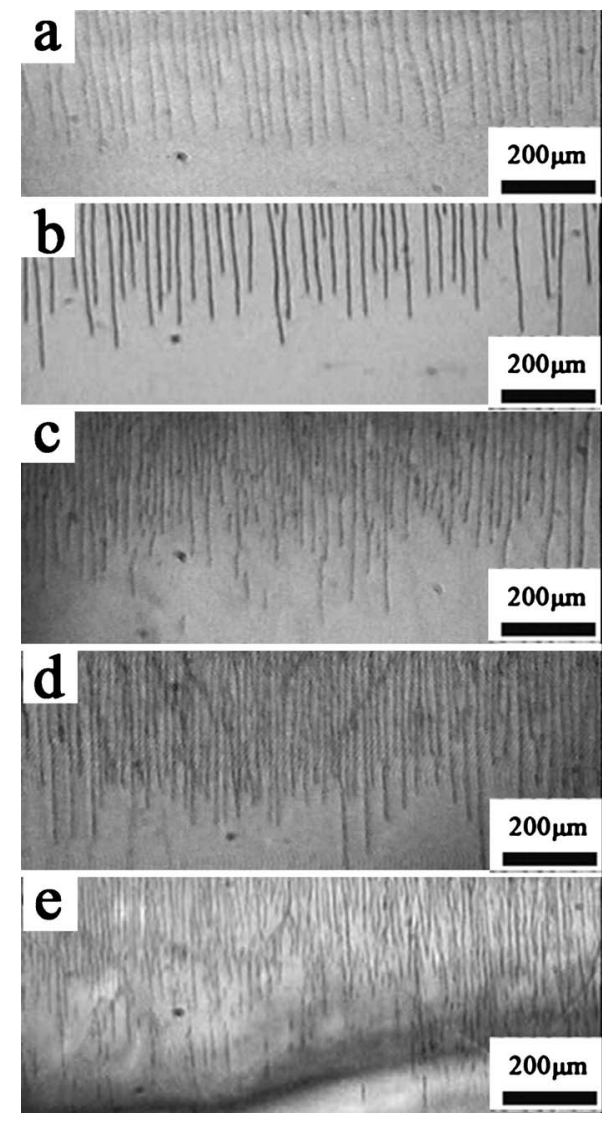

FIG. 3. Optical images of the cracks spacing of the films formed of different radii spheres: (a) $R=85 \mathrm{~nm}$, Cracks spacing W $\approx 15.6 \pm 0.77 \mu \mathrm{m} ;$ (b) $R=110 \mathrm{~nm}, \mathrm{~W} \approx 12.5 \pm 0.55 \mu \mathrm{m} ;$ (c) $R$ $=150 \mathrm{~nm}, \quad W \approx 8.86 \pm 0.42 \mu \mathrm{m} ; \quad(\mathrm{d}) \quad R=180 \mathrm{~nm}, \quad W$ $\approx 8.17 \pm 0.44 \mu \mathrm{m} ;(\mathrm{e}) R=215 \mathrm{~nm}, \mathrm{~W} \approx 7.53 \pm 0.50 \mu \mathrm{m}$.

ticles. It is clear that the vertical fractures are nearly parallel to the growth direction of the colloidal crystal [Fig. 2(b)]. Several minutes later, cracks extended to the bottom of the screen and branched [Fig. 2(c)], with branches distributed randomly between the main cracks. Secondary cracks are unrelated to the propagating drying front and emerge later between the existing cracks. Residual water evaporation and residual stress relief are responsible for the crack branches that formed several minutes after the main cracks.

Figure 3 shows the mean crack front during the colloidal crystal films growth formed of different radii particles. All growth processes were finished at the same temperature and the mean water evaporation rate was about $0.17 \mathrm{~g} / \mathrm{h}$. The cross-sections of five films formed by different radii spheres are shown in Fig. 4, and their thicknesses are nearly the same $(h=6.3 \pm 0.19 \mu \mathrm{m})$. Cracks in the colloidal crystals with different spheres radii came forth along the film length growth direction. Obviously, as the particle radius increased, the crack spacing decreased and cracks appeared to be denser. From in situ observations of the cracking processes in colloidal crystals formed of particles with different radii and similar thicknesses, we derived a relationship between the crack spacing and the particle radius, which is different from other experiments [22,34]. Komatsu and Sasa [34] identified the mechanism of how cracks become regularly arrayed and found that the interval between neighboring cracks $(2 W)$ was proportional to the $2 / 3$ power of the film thickness $(h)$, i.e., $2 \mathrm{~W} \sim E^{-1 / 3} \mathrm{~h}^{2 / 3}$, where $E$ is the Young's modulus of the film if it is independent on the particle radius. Clearly, the crack spacing predicted by Komatsu and Sasa also does not depend on the particle radius, thus does not match our experimental data. Based on Fig. 3, the crack spacing is more sensitive to the particles radius in colloidal film forming from a suspension without gelation.

On the other hand, Lee and Routh [22] concluded that the crack spacing was proportional to the particle radius, i.e.,

$$
\begin{aligned}
2 W \approx & 0.07\left[\frac{20}{75} \sqrt{\frac{3 \gamma_{w a} \eta_{0}}{\dot{E}}} \frac{(1-\Phi)^{2}}{\mu \Phi^{2} h}\right]^{-4 / 5} \\
& \times\left[\frac{20(1-\Phi)^{2}}{75 \mu \Phi^{2}}\left(\frac{3 \eta_{0} \gamma_{w a}^{3}}{\dot{E}^{3}}\right)^{1 / 4}\right] R^{1 / 5},
\end{aligned}
$$

where $2 W$ is the cracks spacing, $\mu$ is water viscosity, $\Phi$ is the solid phase volume fraction, $\eta_{0}$ is the dispersion viscosity, $\gamma_{\text {wa }}$ is water surface tension, $\dot{E}$ is water evaporation rate, $h$ is the dried film thickness, and $R$ is the particle radius. Their experiments were performed in a Petri dish, which is different compared with our CVDD system. Film structure and quality are also different, as uniform film did not form in those experiments [22]. Equation (1) was derived from the experimental data. Thus differences between the two drying systems did not allow our results to match Lee and Routh prediction either. We present theoretical analysis that explains observed cracking phenomena in our CVDD system.

\section{THEORETICAL ANALYSIS OF THE FRACTURE PROCESS}

In this paper, we consider a variable shear modulus $(G)$ that plays an important role in fracture of colloidal crystals formed of viscoelastic or quasielastic spheres. Brown [35],

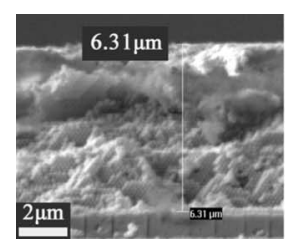

(a)

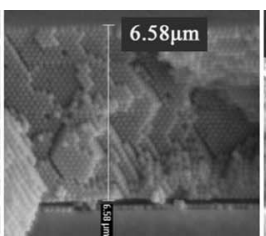

(b)

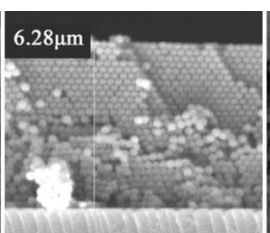

(c)

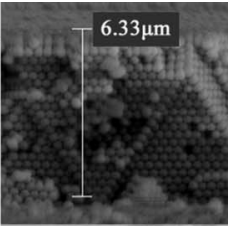

(d)

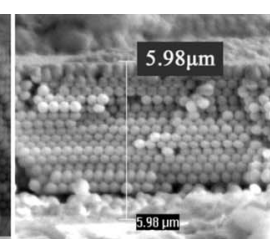

(e)

FIG. 4. Film thickness measured by SEM: (a) $R=85 \mathrm{~nm}, h=6.31 \mu \mathrm{m}$; (b) $R=110 \mathrm{~nm}, h=6.58 \mu \mathrm{m}$; (c) $R=150 \mathrm{~nm}, h=6.28 \mu \mathrm{m}$; (d) $R=180 \mathrm{~nm}, h=6.33 \mu \mathrm{m}$; (e) $R=210 \mathrm{~nm}, h=5.98 \mu \mathrm{m}$. 
Mason [36], Eckersley and Rudin [37] obtained conditions for film formation to occur from different mechanical models, respectively, as $G \leq 35 \gamma_{w a} / R, \quad G \leq 266 \gamma_{w a} / R, \quad G$ $\leq 34 \gamma_{w a} / R$. The real value of the shear modulus during film formation is difficult to be measured. In Ref. [37], Eckersley and Rudin made dynamic mechanical measurements using a dried pressed polymer sample. The experimentally measured shear modulus far exceeded the maximum possible values predicted by theoretical models, but still showed a trend that the film shear modulus decreases with the particle radius. Thus, we assume a special value following the maximum value of Eckersley and Rudin's

$$
G=34 \gamma_{w a} / R
$$

as the variable shear modulus, which can satisfy all film formation conditions mentioned above and follow the trend of the real value of the shear modulus measured in Ref. [37].

Considering the variable shear modulus [Eq. (2)] and the constant film thickness, Komatsu and Sasa's equation can be written as $2 W \sim R^{n}$, where $n>0$. Thus crack spacing $2 W$ increases with particle radius $R$, which does not agree with our experimental data. This may be due to the differences between the two experimental systems. Komatsu and Sasa's equation is derived from Allain and Limat's work. The film undergoes gel formation, thus the volume shrinkage is an important factor in the film deformation and fracture processes. In our experiments the film does not form a gel, thus it is similar to a porous film failure during evaporation. Because of these differences Komatsu and Sasa's equation does not apply in our case.

To explain the periodicity of crack patterns observed, Tirumkudulu and Russel [21] used energy balance analysis to predict crack dynamics for multiple cracks. The total elastic energy, $\Delta E$, (per unit area of the crack surface) for multiple cracks is given as

$$
\begin{aligned}
\Delta E= & -\frac{3}{2} h \sigma_{0} \varepsilon_{0}\left(\frac{1}{\bar{k}}-\frac{\bar{k}}{3}\right)\left\{\tanh \left(\frac{\bar{k} W}{2 h}\right)\right. \\
& \left.-\frac{1-\bar{k}^{2}}{3-\bar{k}^{2}} \cdot \frac{\bar{k} W / 2 h}{\cosh ^{2}(\bar{k} W / 2 h)}\right\}
\end{aligned}
$$

and the stress-strain $\left(\sigma_{0}-\varepsilon_{0}\right)$ separation relationship is given as

$$
\sigma_{0}=\frac{3}{35} M G \Phi_{r c p}\left(\varepsilon_{0}\right)^{2},
$$

where $\bar{k}$ is a constant, $\Phi_{r c p}$ is the solid volume fraction of the close-packed structure, and $M$ is the number of neighbor particles. In order to obtain the relationship between $W$ and $R$, one must get the fracture stress $\sigma_{0}$ by other means. The colloidal films theoretical fracture stress could be estimated based on the theories of crystal fracture. Relationship between the cracks spacing and particle radius cannot be obtained directly from Eqs. (3) and (4) because the fracture stress is also unknown. Another approach is needed to predict the fracture stress.

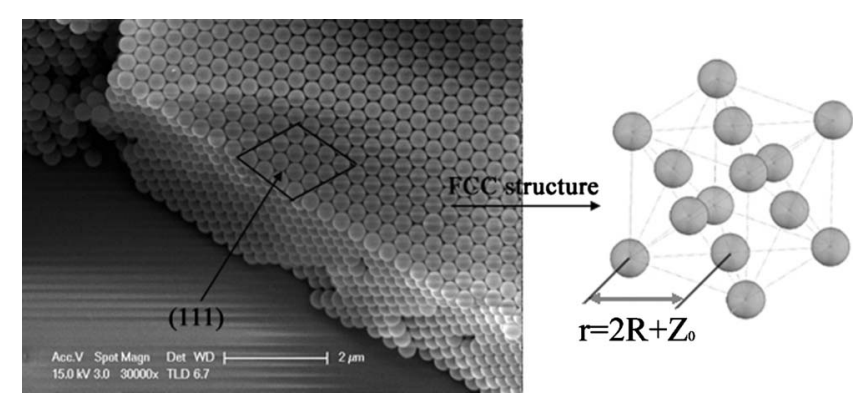

FIG. 5. FCC structure of the film.

Let's consider colloidal particles interactions effects, which include capillary forces, surface tension, tensile stress, gravitational force, electrostatic interactions, and van der Waals forces. Among the interaction forces, capillary force and surface tension are the largest of the external forces that govern film formation [32,33] and particles deformation [10-13]. Tensile stress arises from the internal forces due to particles deformation, but gravitational force, electrostatic repulsion, and van der Waals forces are the ones that keep particles together as a whole solid material, and determine the dried film mechanical properties. Based on the literature results [10-13], gravitational force and electrostatic repulsion in colloid films are much smaller than the van der Waals interactions, thus they can be neglected.

CVDD is one of the most important methods for fabricating 3D colloidal crystals with unique particles arrangement. As a result of the experiments presented in this paper, a series of FCC structured films was made [38]. Thus, we assume that the film cracks along the $(1 \overline{1} 0)$ crystallographic planes, since cracking along a different set of planes will only bring a constant coefficient to our results. Figure 5 shows FCC crystal structure of the film. A unit cell in the film that is under stress $\sigma_{0}$ will have the stored elastic energy of $6 \sqrt{2} \sigma_{0} R^{2} d r$, and the total attractive energy between two surfaces would be $\frac{3}{2} \frac{\partial U}{\partial Z_{0}} d r$. Here, $r$ is the distance between centers of two contact spheres, $\frac{\partial U}{\partial Z_{0}}$ is the van der Waals attractive force between the two contact particles and $Z_{0}$ is the minimal distance between the particles. Thus, the necessary condition for crack propagation is

$$
\sigma_{0}=\frac{\sqrt{2}}{8 R^{2}} \frac{\partial U}{\partial Z_{0}} .
$$

The van der Waals attractive force of two contact particles can be obtained from Ref. [39],

$$
\frac{\partial U}{\partial Z_{0}}=\frac{A}{12 Z_{0}^{2}}\left(R+\frac{a^{2}}{Z_{0}}\right),
$$

where $A$ is the Hamaker constant and $a$ is the deformation contact radius. Eckersley and Rudin [37] predicted particles deformation contact radius $a$ under closed packing conditions:

$$
a^{2}=0.191 R^{2} .
$$

Substitution of Eqs. (6) and (7) into Eq. (5) yields 
TABLE I. Experimental constants used in theoretical analysis.

\begin{tabular}{ccccccc}
\hline \hline$A(J)^{\mathrm{a}}$ & $M^{\mathrm{b}}$ & $\begin{array}{c}\mathrm{Z}_{0} \\
(\mathrm{~nm})^{\mathrm{c}}\end{array}$ & $\Phi_{r c p}$ & $\begin{array}{c}\gamma_{\mathrm{wa}} \\
(\mathrm{N} / \mathrm{m})\end{array}$ & $\begin{array}{c}\bar{h} \\
(\mathrm{~m})\end{array}$ & $\bar{k}^{\mathrm{d}}$ \\
\hline $1.0 \times 10^{-20}$ & 6 & $0.4 \times 10^{-9}$ & 0.74 & 0.073 & $6.3 \times 10^{-6}$ & 0.699 \\
\hline \hline
\end{tabular}

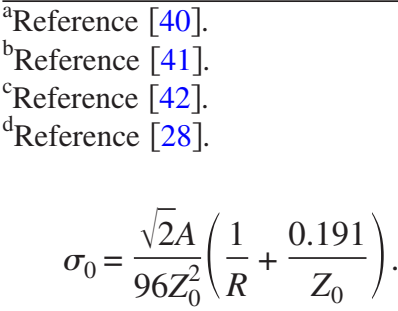

Equation (8) is the theoretical value of the critical stress. The actual stress is always smaller than the theoretical value because of the presence of lattice defects and microcracks between particles, which can significantly decrease the structural strength of the colloidal crystal. It is reasonable to assume that the actual colloid crystal fracture stress will be at least twice less than the theoretical strength. For example, according to Tirumkudulu and Russel's results [Eqs. (3) and (4)] for $110 \mathrm{~nm}$ particles radius, colloid crystal strength would be $1.77 \times 10^{5} \mathrm{~Pa}$. Meanwhile, the theoretical critical stress predicted by our theory is $4.48 \times 10^{5} \mathrm{~Pa}$. Thus, we take

$$
\sigma \approx \frac{1.77}{4.48} \sigma_{0} \approx 5.8 \times 10^{-3} \frac{A}{Z_{0}^{2}}\left(\frac{1}{R}+\frac{0.191}{Z_{0}}\right)
$$

as the real critical stress here.

For equilibrium crack propagation, the total elastic energy recovered must balance the increase in interfacial energy. Since the van der Waals attractive energy per unit area $(\Sigma U)$ is much smaller than the interfacial energy between water and air $\left(\gamma_{\text {wa }}\right)$, i.e., $\Sigma U \ll 2 \gamma_{\text {wa }}$, water surface energy plays the dominating role. The energy balance can be written as

$$
\Delta E+\sum U+2 \gamma_{w a} \approx \Delta E+2 \gamma_{w a}=0 .
$$

Substitution of Eqs. (1)-(3) and (9) into Eq. (10) yields

$$
\begin{aligned}
\Delta E \approx & -2 \gamma_{w a} \approx-3.88 \times 10^{-4} \frac{h A^{3 / 2}}{Z_{0}^{3}\left(\gamma_{w a} M \Phi_{r c p}\right)^{1 / 2}}\left(\frac{1}{\bar{k}}-\frac{\bar{k}}{3}\right) \\
& \times\left\{\tanh \left(\frac{\bar{k} W}{2 h}\right)-\frac{1-\bar{k}^{2}}{3-\bar{k}^{2}} \frac{\bar{k} W / 2 h}{\cosh ^{2}[\bar{k} W / 2 h]}\right\} \\
& \times\left[\frac{1}{R}+\frac{0.191}{Z_{0}}\right]^{3 / 2} \cdot R^{1 / 2} .
\end{aligned}
$$

In order to make Eq. (11) dimensionless, we take $\bar{W}=\frac{\bar{k} W}{2 h}$ and $\bar{R}=\frac{R}{R_{0}}$, where $R_{0}$ is $150 \mathrm{~nm}$ (mean particles radius used in experiments). Constants $Z_{0}, A, M, \Phi_{\text {rcp }}, \bar{k}$, and $\gamma_{\text {wa }}$ are listed in Table I, and the film thickness, $h$, is nearly an invariable number (Fig. 4), thus Eq. (11) turns to be

$$
\begin{aligned}
3.65 & \times 10^{-3}\left\{\tanh \bar{W}-0.204 \frac{\bar{W}}{\cosh ^{2} \bar{W}}\right\} \\
\times & {\left[\frac{1}{\bar{R}}+71.625\right]^{3 / 2} \cdot \bar{R}^{1 / 2}=1 }
\end{aligned}
$$

in the normalized form.

Figure 6 shows theoretical relationship between $\bar{W}$ and $\bar{R}$ from Eq. (12). Points a-e are experimental values for 85 , $110,150,180$, and $250 \mathrm{~nm}$ particles radii, respectively. It is clear that $\bar{W}$ is a reciprocal ratio to $\bar{R}$, while this effect gets weaker as the particle radius is gradually increased. It is evident that the estimation from Eq. (12) is close to the experimental data, indicating that the empirical equations formulated in this paper can be used to predict the relationship between crack spacing and particle radius of single crystal film with FCC structure formed by the CVDD method. The agreement also suggests that the assumptions used to obtain Eqs. (11) and (12) from Eq. (2) are reasonable, and the variable shear modulus indeed plays an important role in fracture of colloidal crystals formed without gelation.

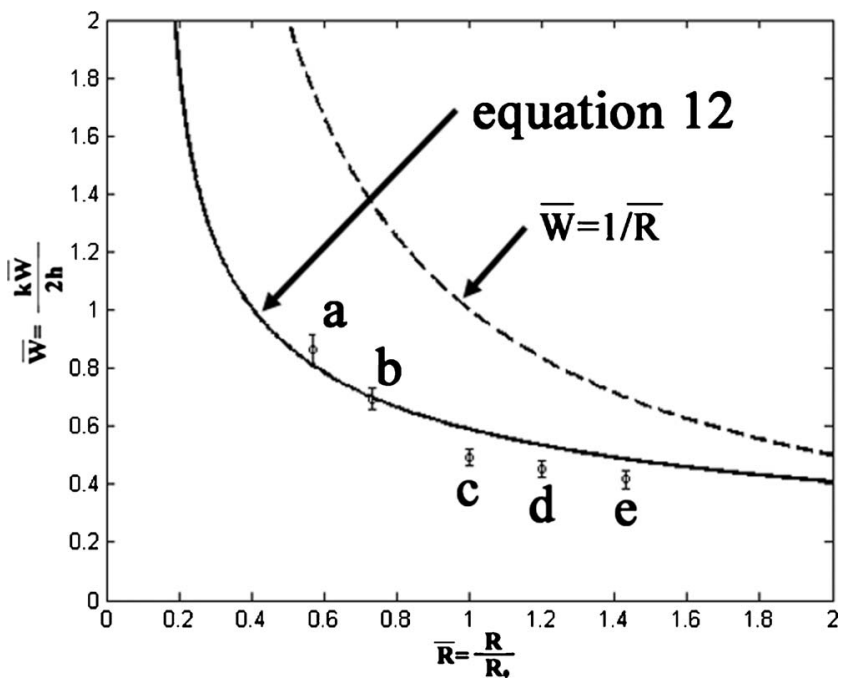

FIG. 6. Theoretical relationship between $\bar{W}$ and $\bar{R}$. Points a-e are the experimental values for $85,110,150,180$, or $215 \mathrm{~nm}$ particles radii, respectively. (a) $\bar{R}=0.57, \bar{W}=0.87 \pm 0.05$; (b) $\bar{R}=0.73$, $\bar{W}=0.69 \pm 0.04 ; \quad$ (c) $\bar{R}=1.00, \quad \bar{W}=0.49 \pm 0.03 ; \quad$ (d) $\bar{R}=1.20, \quad \bar{W}$ $=0.45 \pm 0.03$; (d) $\bar{R}=1.43, \bar{W}=0.42 \pm 0.03 .1 / \bar{R}$ trend line is added for comparison. 


\section{CONCLUSIONS}

In conclusion, controlled vertical drying deposition of solid films formed of polystyrene latex spheres was studied. Real-time observations of crack growth indicate that the cracks extend along with the film vertical growth direction. As water evaporation continues after the film formation, vertical cracks run parallel because of the stress gradient, which points outwards from the liquid region. Then later, secondary cracks appear between the existing cracks, caused by the system self-aggregation, residual water evaporation and stress relaxation.

Based on the force analysis, it was concluded that the film strength arises from the van der Waals forces acting among the particles. For longitudinal cracks, the former conclusions along with the crystal fracture theory were utilized to provide theoretical analysis of the film fracture. As a result, Eq. (11) was derived, and includes major factors, such as film thick- ness, particle radius, water surface tension and the film structure. Finally, Eq. (11) was presented in the dimensionless form to predict the crack spacing dependence on the particle radius [Eq. (12)]. The dimensionless crack spacing $\bar{W}$ is a reciprocal ratio of the dimensionless particle radius $\bar{R}$, and this effect gets weaker as the normalized particle radius is gradually increased, which shows an excellent agreement with experimental observations. Variable shear modulus plays an important role in fracture of colloidal crystals formed without gelation.

\section{ACKNOWLEDGMENTS}

This project was supported by the National Natural Science Foundation of China (Grant No. 10832011), the Knowledge Innovation Program of the Chinese Academy of Sciences (Grant No. KJCX2-YW-L08) and the National Science Foundation under Grant No. CMMI-0600266.
[1] E. Yablonovitch, Phys. Rev. Lett. 58, 2059 (1987).

[2] S. John, Phys. Rev. Lett. 58, 2486 (1987).

[3] O. Painter, R. K. Lee, A. Scherer, A. Yariv, J. D. O'Brien, P. D. Dapkus, and I. Kim, Science 284, 1819 (1999).

[4] R. F. Cregan, B. J. Mangan, J. C. Knight, T. A. Birks, P. St. J. Russell, P. J. Roberts, and D. C. Allan, Science 285, 1537 (1999).

[5] P. Jiang, F. Bertone, K. S. Hwang, and V. L. Colvin, Chem. Mater. 11, 2132 (1999).

[6] A. Imhof and D. J. Pine, Nature (London) 389, 948 (1997).

[7] H. H. Wickman and J. N. Korley, Nature (London) 393, 445 (1998).

[8] Z. D. Cheng, W. B. Russel, and P. M. Chaikin, Nature (London) 401, 893 (1999).

[9] D. Mei, H. Liu, B. Cheng, Z. Li, D. Zhang, and P. Dong, Phys. Rev. B 58, 35 (1998).

[10] A. Toussaint and M. D. Wilde, Prog. Org. Coat. 30, 113 (1997).

[11] M. Visschers, L. Laven, and R. V. Linde, Prog. Org. Coat. 31, 311 (1997)

[12] P. A. Steward, J. Hearn, and M. C. Wilkinson, Adv. Colloid Interface Sci. 86, 195 (2000).

[13] M. Visschers, J. Laven, and A. L. German, Prog. Org. Coat. 30, 39 (1997).

[14] Z. Y. Li and Z. Q. Zhang, Phys. Rev. B 62, 1516 (2000).

[15] A. Hartsuiker and W. L. Vos, Langmuir 24, 4670 (2008).

[16] A. A. Chabanov, Y. Jun, and D. Norris, Appl. Phys. Lett. 84, 3573 (2004).

[17] A. T. Skjeltorp and P. Meakin, Nature (London) 335, 424 (1988).

[18] C. Allain and L. Limat, Phys. Rev. Lett. 74, 2981 (1995).

[19] L. Pauchard, F. Parisse, and C. Allain, Phys. Rev. E 59, 3737 (1999).

[20] K. A. Shorlin, J. R. de Bruyn, M. Graham, and S. W. Morris, Phys. Rev. E 61, 6950 (2000).
[21] M. S. Tirumkudulu and W. B. Russel, Langmuir 21, 4938 (2005).

[22] W. P. Lee and A. F. Routh, Langmuir 20, 9885 (2004).

[23] E. R. Dufresne, E. I. Corwin, N. A. Greenblatt, J. Ashmore, D. Y. Wang, A. D. Dinsmore, J. X. Cheng, X. S. Xie, J. W. Hutchinson, and D. A. Weitz, Phys. Rev. Lett. 91, 224501 (2003).

[24] J. Frenkel, J. Phys. (USSR) 9, 385 (1943).

[25] H. Herz, J. Reine Angew. Math. 92, 156 (1881).

[26] K. L. Johnson, K. Kendell, and A. D. Robert, Proc. R. Soc. London, Ser. A 324, 301 (1971).

[27] B. V. Derjaguin, V. M. Muller, and Y. P. Toporov, J. Colloid Interface Sci. 53, 314 (1975).

[28] A. F. Routh and W. B. Russel, Langmuir 15, 7762 (1999).

[29] K. Chen, A. Taflove, Y. L. Kim, and V. Backman, Appl. Phys. Lett. 86, 033101 (2005).

[30] G. Gauthier, V. Lazarus, and L. Pauchard, Langmuir 23, 4715 (2007).

[31] R. D. Deegan, O. Bakajin, T. F. Dupont, G. Huber, S. R. Nagel, and T. A. Witten, Nature (London) 389, 827 (1997).

[32] N. D. Denkov, O. D. Velev, P. A. Kralchevsky, I. B. Ivanov, H. Yoshimura, and K. Nagayama, Nature (London) 361, 26 (1993).

[33] A. S. Dimitrov and K. Nagayama, Langmuir 12, 1303 (1996).

[34] T. S. Komatsu and S. I. Sasa, J. Appl. Phys. 36, 391 (1997).

[35] G. L. Brown, J. Polym. Sci. 22, 423 (1956).

[36] G. Mason, Br. Polym. J. 5, 101 (1973).

[37] S. T. Eckersley and A. Rudin, J. Coat. Technol. 62, 89 (1990).

[38] D. Lan, Y. R. Wang, Y. M. Zhang, and Z. H. Wu, Chin. Phys. C 33, 1016 (2009).

[39] H. Y. Xie, Powder Technol. 94, 99 (1997).

[40] J. Israelachvili, Intermolecular and Surface Forces (Academic Press, Amsterdam, 1991).

[41] A. Donev et al., Science 303, 990 (2004).

[42] H. Krupp, Adv. Colloid Interface Sci. 1, 111 (1967). 